\title{
Impact of Diurnal Periodicity, Temperature, and Light on Sporulation of Bremia lactucae
}

\author{
Berit Nordskog, David M. Gadoury, Robert C. Seem, and Arne Hermansen
}

First author: Norwegian University of Life Sciences, Department of Plant and Environmental Sciences, N-1432 Ås, Norway; second and third authors: Department of Plant Pathology, Cornell University, New York State Agricultural Experiment Station, Geneva 14456; and fourth author: Bioforsk-Norwegian Institute for Agricultural and Environmental Research, Plant Health and Plant Protection Division, Høgskoleveien 7, N-1432 Ås, Norway.

Accepted for publication 3 April 2007.

\begin{abstract}
Nordskog, B., Gadoury, D. M., Seem, R. C., and Hermansen, A. 2007. Impact of diurnal periodicity, temperature, and light on sporulation of Bremia lactucae. Phytopathology 97:979-986.

We evaluated direct and interactive effects of light quality and intensity, temperature and light, diurnal rhythms, and timing of high relative humidity during long day lengths on sporulation of Bremia lactucae, the causal agent of lettuce downy mildew, using inoculated lettuce seedlings and detached cotyledons. Suppression of sporulation by light was strongly dependent upon temperature and there was little suppression at $\leq 10^{\circ} \mathrm{C}$.

sporulation by light, a clear diurnal pattern of sporulation was observed independent of light and darkness. This diurnal rhythm potentially could interact with light and temperature to confound the results of controlled environment studies, and may be the controlling factor in timing of sporulation at low temperatures. Forecasting models that currently use sunrise and sunset to delimit periods conducive to sporulation can be adapted to short nights and extended twilight conditions by incorporating the effects reported herein. Additionally, models of sporulation could be adapted to better reflect a decrease or absence of the suppressive effect of light at $<15^{\circ} \mathrm{C}$.
\end{abstract} The most suppressive waveband was in the range from 400 to $450 \mathrm{~nm}$, although a lesser effect of wavebands from 450 to 500 and 500 to $550 \mathrm{~nm}$ could be detected. At $15^{\circ} \mathrm{C}$, near the lower threshold for suppression of
Additional keywords: circadian rhythm.
Lettuce downy mildew, caused by Bremia lactucae Regel, represents a major constraint in lettuce production worldwide. Severe outbreaks of the disease usually are sporadic and closely associated with specific environmental conditions. Consequently, several researchers have investigated the impact of various environmental factors in an attempt to better understand the epidemiology of this pathogen, and to decide how and when control measures will be most effective. Environmental factors such as light, temperature, relative humidity $(\mathrm{RH})$, wind, and vibration have been reported to influence spore production, survival, and dispersal for downy mildews $(4,12,22,23,26)$. With the exception of Su et al. $(22,23)$, who studied the combined effects of light and $\mathrm{RH}$ on spore release (22), and of $\mathrm{RH}$ and temperature and $\mathrm{RH}$ and wind speed in relation to sporulation (23), and Cohen and Eyal (4), who studied the interaction of light and temperature on growth and differentiation of sporangiophores and sporangia, the foregoing factors have been studied singly and in isolation, excluding possible interaction (e.g., between light and temperature), despite the fact that spore dispersal under field conditions often takes place while the above factors vary simultaneously.

Light has been reported to operate as a limiting factor for sporulation and spore survival and a trigger for spore release of many downy mildews when the other environmental factors are favorable $(2,4,6,8,19,21,22,25,27)$. However, it is unclear from previous studies whether the noted effects were due to a direct

Corresponding author: D. M. Gadoury; E-mail address: dmg4@ nysaes.cornell.edu

*The $\boldsymbol{e}$-Xtra logo stands for "electronic extra" and indicates that the online version contains supplemental material not included in the print edition. Figure 8 appears in color online.

doi:10.1094/PHYTO-97-8-0979

(c) 2007 The American Phytopathological Society suppressive effect of light or a diurnal periodicity in which sporulation does not occur during an interval coincident with normal daylight hours. Verhoeff (25) reported that continuous light would retard but not completely suppress sporulation of B. lactucae on lettuce plants when incubated in high humidity overnight. Subsequently, Raffray and Sequeira (19) reported that maximum sporulation of B. lactucae was obtained after $6 \mathrm{~h}$ of darkness, but some sporulation occurred after as little as $2 \mathrm{~h}$ of darkness. They also suggested that continuous light affected penetration and establishment of the pathogen during the first $24 \mathrm{~h}$ after inoculation but did not seem to affect development of the pathogen thereafter. Again, it is not known whether these responses are directly related to light or a diurnal response in the host plant or in the pathogen itself, because circadian rhythms have been described for both plants (24) and fungi (1).

Where light is directly involved in regulation of sporulation, it is plausible that a threshold intensity or dose must be reached for a detectable effect. Brook (2) reported that the suppressive effect of light on sporulation in Plasmopara viticola was greatest at intensities exceeding 3 or $3.5 \mathrm{Wm}^{-2}$ for wavelengths in the near$\mathrm{UV}$ or green range, respectively. There are no reports of the minimum intensity of light associated with suppressed sporulation of B. lactucae; however, an intensity of $8 \mathrm{Wm}^{-2}$ or $41 \mu \mathrm{mol} \mathrm{s}{ }^{-2} \mathrm{~m}^{-2}$ has been associated with spore release (26). It is not clear whether the purported effect of light is exerted by a cumulative irradiation dose, or a critical intensity, or whether the effect is exerted across a broad spectral range or only by specific wavelengths.

The reported effect of light quality on sporulation of downy mildews has varied. Although Brook (2) reported that near-UV and green light were the most suppressive toward sporulation of P. viticola, Rumboltz et al. (20) reported that the blue range of the spectrum was the most suppressive to spore production. In B. lactucae, Raffray and Sequeira (19) reported that sporulation 
was inhibited equivalently in both unfiltered light and green light. Cohen and Eyal (4) reported an inhibitory effect of blue light on sporulation in Pseudoperonospora cubensis. The spectral selectivity and precise spectral distributions of the lighting systems
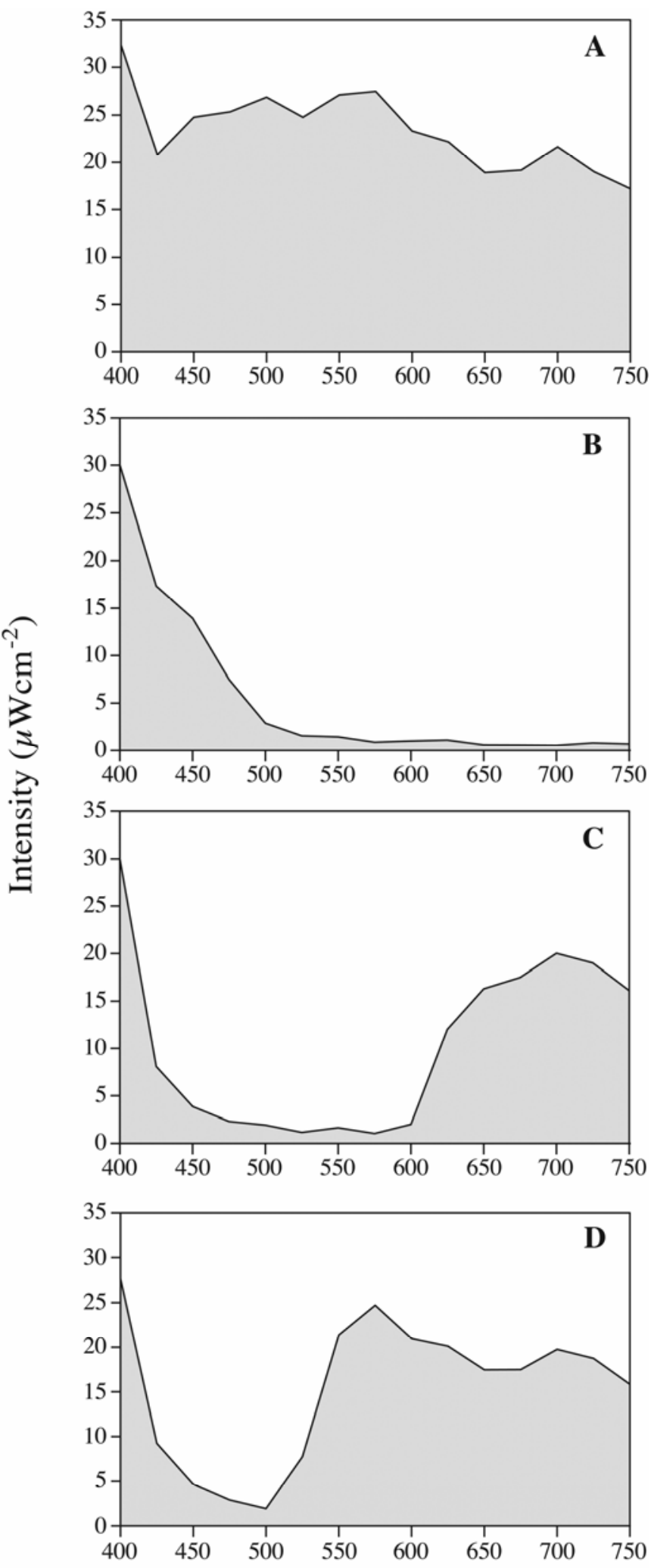

\section{Wavelength (nm)}

Fig. 1. Spectral distributions of light from a $150-\mathrm{W}$ halogen source in conjunction with $\mathbf{A}$, a daylight-balanced filter and $\mathbf{B}$, violet; $\mathbf{C}$, red; and $\mathbf{D}$, yellow wide-bandpass filters as measured with a multispectral radiometer. (both filters and source) used in the above studies were not defined for all reports on the effects of light.

Despite some imprecision and uncertainty in defining the direct effect of light, it is a common component of forecasting models for many downy mildews $(10,11,13,14,17)$ and, specifically, for downy mildew of lettuce $(3,22,26)$. At Nordic latitudes, day length, light intensity, and light quality differ substantially from most other lettuce-production regions of the world. Therefore, the specific effects of light are of great interest in evaluating forecasting models for B. lactucae. Our objectives were to precisely evaluate the direct effects of light intensity and quality on spore production of $B$. lactucae, to elucidate how temperature might modify the effect of light on spore production, and to investigate the possible involvement of diurnal rhythms as an additional factor affecting spore production. Preliminary reports have been published $(15,16)$.

\section{MATERIALS AND METHODS}

Maintenance of host plants and pathogen isolates. Seed of lettuce cv. Black Seeded Simpson were planted in soil (Miracle Grow Lawn Products Inc., Marysville, OH; or P-jord, Emmaljunga Torvmull AB, Vittsjö, Sweden) contained in 9-cm glass petri dishes. Emergent seedlings were grown for 7 days at 20 to $22^{\circ} \mathrm{C}$ with a 16-h photoperiod (light intensity of $55 \mu \mathrm{mol} \mathrm{m} \mathrm{m}^{-2} \mathrm{~s}^{-1}$ ) until cotyledons were fully expanded. Seedlings were inoculated by spraying them with a spore suspension prepared as follows. Lettuce tissue bearing sporulating lesions was added to a $50-\mathrm{ml}$ centrifuge tube containing distilled water and shaken by hand. The tissue then was removed and the resultant suspension was diluted to a concentration of $3 \times 10^{5}$ spores $/ \mathrm{ml}$. Seedlings were sprayed until runoff and incubated wet overnight at $15^{\circ} \mathrm{C}, 100 \%$ $\mathrm{RH}$, and $8 \mathrm{~h}$ of darkness to allow infection. The seedlings then were kept at 20 to $22^{\circ} \mathrm{C}, 70 \% \mathrm{RH}$, and light conditions as above during the following 6 days to prevent sporulation before commencement of experiments. Experiments were conducted using an isolate of B. lactucae collected in June 2003 from field-grown lettuce in Oswego, NY (NY-1) or an isolate collected in July 2003 in Lier, Buskerud County in Norway (NOR-1).

Direct and interactive effects of light and temperature. Lettuce seedlings were inoculated with isolate NY-1 and incubated at 20 to $22^{\circ} \mathrm{C}$ and $70 \% \mathrm{RH}$ for six nights as above to suppress sporulation. Experiments then were initiated in a nested design with three replicate groups of seedlings in separate $9-\mathrm{cm}$ petri dishes with soil for each treatment. On the evening of the sixth day, the seedlings were incubated at $100 \% \mathrm{RH}$ from 2200 to $0800 \mathrm{~h}$ in five growth chambers (main plots) at temperatures of 5, 10, 15, 20 , and $25^{\circ} \mathrm{C}$ in either darkness or under illumination (GE F20T12 PL/AQ Wide spectrum; General Electric, Fairfield, CT) at an intensity of $40 \mu \mathrm{mol} \mathrm{m} \mathrm{m}^{-2} \mathrm{~s}^{-1}$ (subplots). The following morning, $\geq 20$ cotyledons supporting sporulation were selected arbitrarily and detached from each group of seedlings. Spores were rinsed from each group of cotyledons into a 5-ml volume of distilled water and were enumerated with a hemacytometer at $\times 200$. Results were expressed as spores per cotyledon. The experiment was repeated three times. The interactive effects of temperature and light on the number of spores per cotyledon were analyzed by analysis of variance (ANOVA) using the Proc GLM procedure with the effect of light nested within temperature (SAS Institute, Cary, NC). The effect of temperature on number of spores produced per cotyledon in darkness was analyzed using Tukey's family error rate at $95 \%$ significance level, whereas the suppressive effect of light in relation to temperature was analyzed using linear regression analysis (Minitab 14.1; MiniTab Inc., State College, PA).

Effects of light intensity and quality on sporulation. In order to uniformly and consistently supply light of controlled quality and intensity to the lower leaf surface, lettuce seedlings were 
raised and inoculated with isolate NY-1 or NOR-1 as described above, and then the cotyledons were detached and placed with the abaxial surface uppermost on wet filter paper in open petri dishes on the sixth night after inoculation. Treatments were replicated three times using 10 cotyledons per replicate. The open petri dishes containing the cotyledons were transferred to $15-$ to $18-\mathrm{cm}-$ diameter capped cylinders and were maintained at $20^{\circ} \mathrm{C}$ and $100 \% \mathrm{RH}$ overnight to promote sporulation. When required, light was supplied to the interior of a cylinder through a fiberoptic bundle connected to a 150-W quartz-halogen illuminator described in a previous study (9). The fiberoptic bundle was inserted at the top of the vertical cylinder at a distance of $20 \mathrm{~cm}$ above the cotyledons. Quality of light (Figs. 1 and 2) was regulated by inserting wide-bandpass (Dolan-Jenner Industries, Inc., Woburn, MA) or narrow-bandpass filters (3rd Millennium; Omega Optical Inc., Brattleboro, VT) into the light path at the source and was measured at the sample surface using a multispectral radiometer as described by Gadoury et al. (9). The cotyledons were incubated for $10 \mathrm{~h}$ overnight at $20^{\circ} \mathrm{C}$ and $100 \% \mathrm{RH}$ while exposed to different qualities of light, including a dark control and a light control. Light intensities for wide-bandpass filters in combination with a daylight-balanced filter used for isolate NY-1 were 8.4, 4.7, and $2.0 \mu \mathrm{mol} \mathrm{m} \mathrm{m}^{-2} \mathrm{~s}^{-1}$ for the yellow, red, and violet filters, respectively, as measured with a Li-Cor Quantum meter (LI250A; Li-Cor Biosciences, Lincoln, NE). The experiment was repeated three times for each of the wide-bandpass filters. Light intensities provided through the narrow-bandpass filters of 400 to 450,450 to 500 , and 500 to $550 \mathrm{~nm}$ were $2.8,1.5$, and $1.3 \mu \mathrm{mol} \mathrm{m}{ }^{-2}$ $\mathrm{s}^{-1}$, respectively for NY-1 as measured with a LI-COR Quantum meter and 6, 7, and $13 \mu \mathrm{mol} \mathrm{m} \mathrm{m}^{-2} \mathrm{~s}^{-1}$, respectively, for NOR-1 as measured with an Apogee Quantum meter (QMSW-SS; Apogee Instruments Inc. Logan, UT). Daylight-balanced light controls for NY-1 were exposed to a light intensity of $13 \mu \mathrm{mol} \mathrm{m} \mathrm{m}^{-2} \mathrm{~s}^{-1}$ (Li-Cor), whereas unfiltered light controls for NOR-1 were exposed to a light intensity of $90 \mu \mathrm{mol} \mathrm{m} \mathrm{m}^{-2} \mathrm{~s}^{-1}$ (Apogee). The experiments with narrow-bandpass filters were repeated three times for each filter and isolate, with the exception of NY-1, where 500 to $550 \mathrm{~nm}$ was repeated twice.

Different intensities of daylight-balanced light were provided by regulating the intensity of the illuminator and by inserting neutral density filters (Omega Optical Inc.) into the light path. Detached cotyledons were incubated for $10 \mathrm{~h}$ overnight, as above, while exposed to different intensities of daylight-balanced light, including a dark control. Isolate NY-1 was tested in light intensities ranging from 4 to $13 \mu \mathrm{mol} \mathrm{m} \mathrm{m}^{-2} \mathrm{~s}^{-1}$, as measured with a LiCor Quantum meter, whereas isolate NOR-1 was tested at light intensities ranging from 1 to $43 \mu \mathrm{mol} \mathrm{m} \mathrm{m}^{-2} \mathrm{~s}^{-1}$, as measured with an Apogee Quantum meter. For all replicates in which $>3$ of 10 inoculated cotyledons supported sporulation, spores were rinsed off the cotyledons and enumerated as previously described. Response to treatments was recorded as percentage of sporulation relative to the dark control.

The suppressive effects of the different light qualities were analyzed by using Dunnett's family error rate at 5\% compared with the dark control. The effects of light intensities were analyzed using linear regression analysis.

Effect of diurnal rhythm on sporulation. Two temporally offset light regimes were used to produce diseased lettuce seedlings with different diurnal rhythms. First, lettuce seed were planted and maintained in growth chambers at $15^{\circ} \mathrm{C}$ and $70 \% \mathrm{RH}$ and provided with $12 \mathrm{~h}$ of light, beginning at either $0900 \mathrm{~h}$ (daytime light) or $2100 \mathrm{~h}$ (night-time light). Light intensity and light source were the same as described for the light and temperature experiments. The seedlings then were inoculated with isolate NY-1 after 7 days, as described above, and kept at $15^{\circ} \mathrm{C}$ and their respective daylight regimes for an additional 7 days. Seedlings from each group then were incubated at $100 \% \mathrm{RH}$ for $12 \mathrm{~h}$ in either light or darkness starting at 0900 and $2100 \mathrm{~h}$. The four treatments were as follows: (i) darkness during the expected night interval (e.g., darkness starting at $2100 \mathrm{~h}$ for seedlings previously conditioned to darkness starting at $2100 \mathrm{~h}$ ), (ii) light during the expected night interval, (iii) darkness during the expected day interval, and (iv) light during the expected day interval. Three replicate groups of seedlings in separate petri dishes with soil were used for each treatment, and spores were harvested and enumerated as previously described from up to 20 cotyledons that were selected arbitrarily from each group of seedlings. The experiment was repeated three times. The data were analyzed by ANOVA using the Proc GLM procedure.

Impact of timing of the period of high RH during long day lengths. Lettuce seed were planted and maintained in growth chambers at $15^{\circ} \mathrm{C}$ and $70 \% \mathrm{RH}$ as for the experiments on diurnal rhythm, but were exposed to the following light regimes: (i) continuous light or (ii) $3 \mathrm{~h}$ of dark beginning at $0900 \mathrm{~h}$. Seedlings
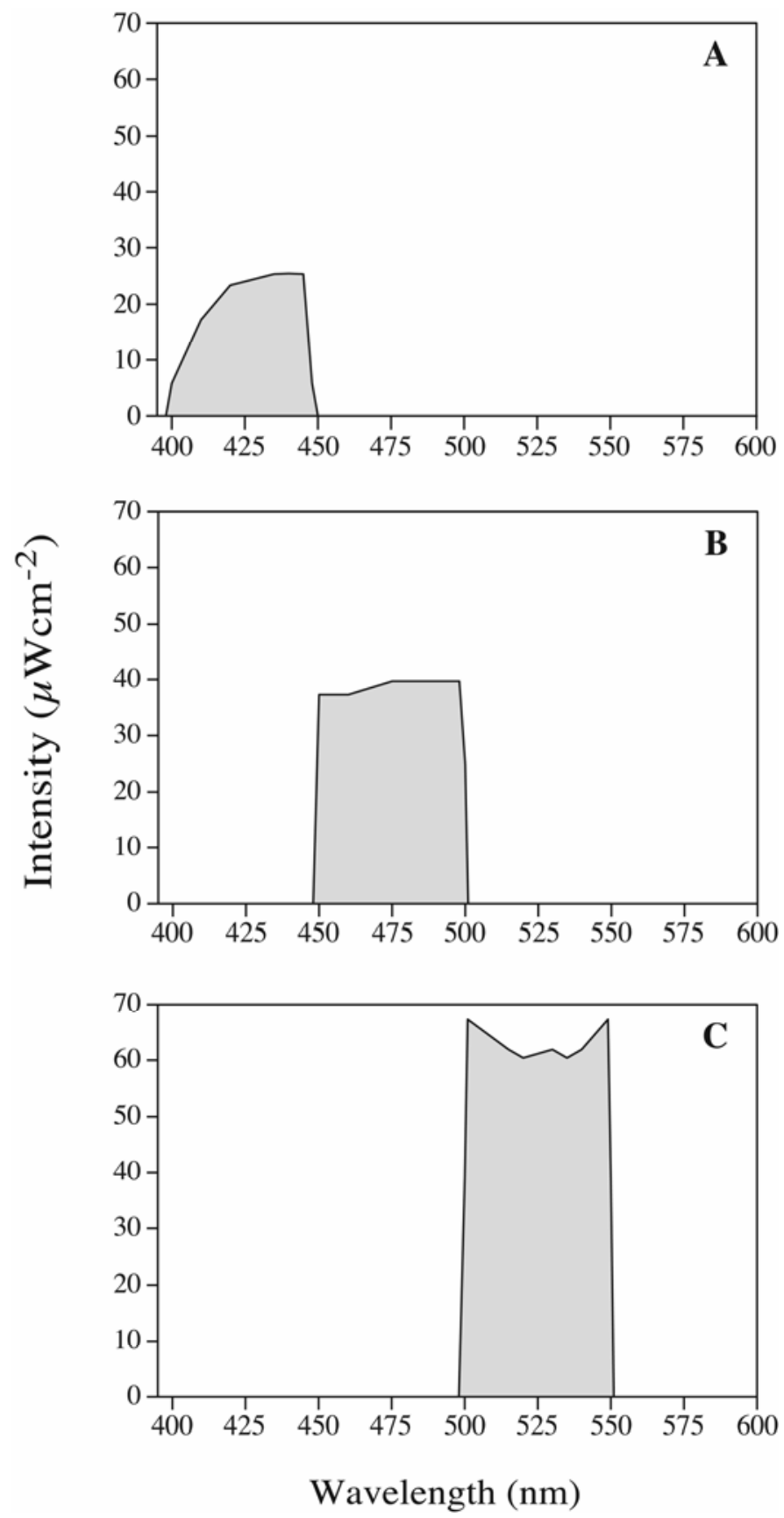

Fig. 2. Spectral distributions of light from a $150-\mathrm{W}$ halogen provided through narrow bandpass filters in the range of $\mathbf{A}, 400$ to $450 ; \mathbf{B}, 450$ to 500 ; and $\mathbf{C}$, 500 to $550 \mathrm{~nm}$ as measured with a multispectral radiometer. 
were inoculated after 1 week with the isolate NY-1 and, 7 days after inoculation, the seedlings were incubated at $15^{\circ} \mathrm{C}$ and $100 \%$ $\mathrm{RH}$ in darkness for $4 \mathrm{~h}$, starting at $0800,0900,1000$, and $1100 \mathrm{~h}$ (i.e., for (ii) above, $1 \mathrm{~h}$ before the start of the dark period, at the start of the dark period, and 1 and $2 \mathrm{~h}$ after the start of the dark period, respectively). At the end of each incubation period, up to 20 cotyledons were collected arbitrarily from each of three replicated groups of seedlings in separate petri dishes; soil and spores were harvested and enumerated as previously described. The experiment was repeated three times. The effects of incubation periods were analyzed by ANOVA, as above.

Germination percentages of spores produced in light and darkness. Detached cotyledons from seedlings inoculated with isolate NY-1 were incubated overnight at $20^{\circ} \mathrm{C}$ and $100 \% \mathrm{RH}$ with the abaxial surface uppermost in (i) complete darkness or (ii)

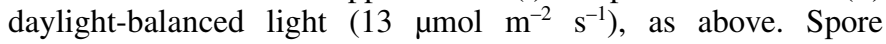
suspensions were prepared from 10 cotyledons for each treatment as described earlier. Droplets of the suspensions were incubated on glass slides at $15^{\circ} \mathrm{C}$ for $4 \mathrm{~h}$ and the slides were examined at $\times 200$. At least 160 spores from the dark treatment and at least 50 spores from the light treatment were classified as germinated or ungerminated based upon the presence of a germ tube at least one-half the diameter of the spore. The experiment was repeated 10 times. The treatment effects on germination were analyzed by ANOVA, as above.

\section{RESULTS}

Direct and interactive effects of light and temperature. ANOVA indicated no significant effect of experimental repeat $(P=0.05)$, and homogeneity of variance allowed data from the three repeats of the experiment to be pooled. There was a significant effect of temperature $(P<0.005)$ and a significant interaction between temperature and light $(P<0.005)$. The number of spores produced during darkness did not differ significantly $(P=0.05)$ at 5,10 , or $15^{\circ} \mathrm{C}$, but declined significantly at 20 and $25^{\circ} \mathrm{C}$ from the maximum observed at $15^{\circ} \mathrm{C}$ (Fig. 3). The suppressive effect of light upon sporulation was directly proportional to temperature between 5 and $25^{\circ} \mathrm{C}$ (Fig. 4). Although presence of light reduced sporulation by nearly $100 \%$ at $25^{\circ} \mathrm{C}$, sporulation in light at 5 or $10^{\circ} \mathrm{C}$ was reduced by $<20 \%$ compared with sporulation in complete darkness (Fig. 4).

Effects of light intensity and quality on sporulation. When light was supplied through wide-bandpass filters, the reduction of sporulation relative to the dark control varied with the experimental repeat. For the experiments using narrow-bandpass filters, ANOVA indicated no significant effect of experimental repeat $(P=0.05)$, and homogeneity of variance allowed data from the three repeats of the experiment to be pooled. Daylight-balanced

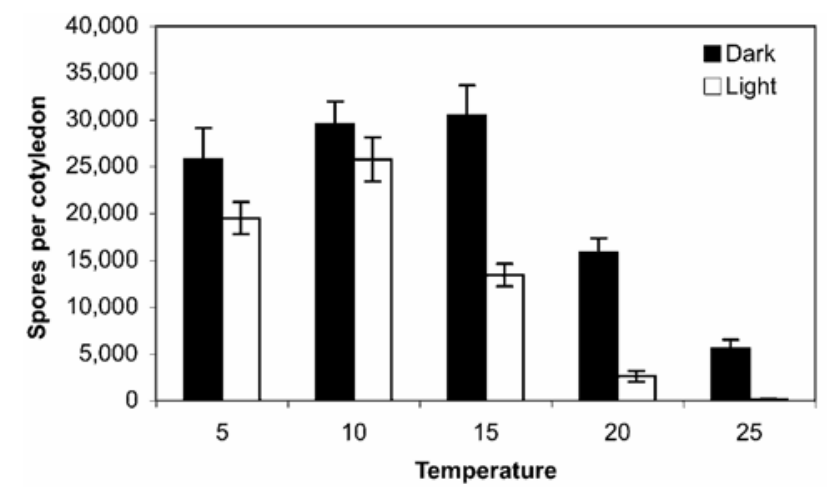

Fig. 3. Interaction plot on effects of temperature and light on the production of spores by Bremia lactucae NY-1. Lettuce seedlings were incubated for $10 \mathrm{~h}$ in either darkness or light $\left(40 \mu \mathrm{mol} \mathrm{m} \mathrm{m}^{-2} \mathrm{~s}^{-1}\right)$ and $100 \%$ relative humidity. Bars indicate one standard error of the mean. light, with a base intensity of $13 \mu \mathrm{mol} \mathrm{m} \mathrm{m}^{-2} \mathrm{~s}^{-1}$, supplied through wide-bandpass filters caused a reduction in sporulation relative to the dark control ranging from $\approx 0$ to $50 \%$ and 0 to $30 \%$ in the red and yellow range, respectively, for isolate NY-1. Light supplied through a wide-bandpass filter in the violet range suppressed sporulation by $\approx 30$ to $60 \%$ relative to the dark control (Fig. 5), providing a more consistent reduction in sporulation relative to the suppression of light in the yellow and red range. However, when light in the violet range of the spectrum was further divided by using narrow-bandpass filters of 50-nm steps, suppression of sporulation for NY-1 was not observed at 400 to $450 \mathrm{~nm}$ (confidence interval $[\mathrm{CI}]=-14.19$ to 13.74 ), significantly reduced at 450 to $500 \mathrm{~nm}(\mathrm{CI}=-38.95$ to -9.68$)$, and nearly significant at 500 to $550 \mathrm{~nm}(\mathrm{CI}=-32.26$ to 0.47 ) (Fig. 6A), indicating a possible effect of light intensity. The suppression of sporulation was resolved for isolate NOR-1 when the base light intensity was increased to $90 \mu \mathrm{mol} \mathrm{m} \mathrm{m}^{-2} \mathrm{~s}^{-1}$, whereupon light in the range of 400 to $450 \mathrm{~nm}$ caused the greatest $(\mathrm{CI}=-102.70$ to -37.59$)$ reduction of sporulation (Fig. 6B), although a lesser effect of the wavebands from 450 to $500 \mathrm{~nm}(\mathrm{CI}=-66.32$ to -1.21$)$ and 500 to $550 \mathrm{~nm}$ (CI $=-54.47$ to 10.63 ) still could be detected (Fig. 6).

Conclusions about the effect of light intensity cannot be drawn from these experiments alone, because the effects of light intensity and pathogen isolate are confounded. However, a similar impact of lower versus higher light intensity was observed for both isolates when $\mathrm{RH}$ and temperature were held constant and isolates were exposed to daylight-balanced illumination. Increasing source intensity was associated with increased uniformity of suppression of sporulation across a range of light intensity. Complete suppression of sporulation was not reached at any light intensity tested and some mature spores were produced at all light intensities (Fig. 7).

Effect of diurnal rhythm on sporulation. ANOVA indicated no significant effect of experimental repeat $(P=0.05)$, and homogeneity of variance allowed data from the three repeats of the experiment to be pooled. A diurnal rhythm operated independently of a suppressive effect of light. When exposure to high RH coincided with an expected "night" interval (based upon the regime of the previous 14 days), sporulation occurred regardless of the presence of light or darkness (Table 1). When inoculated lettuce plants incubated at $15^{\circ} \mathrm{C}$ were conditioned to expect night to begin at $2100 \mathrm{~h}$, exposure to light at an intensity of $40 \mu \mathrm{mol} \mathrm{m}{ }^{-2}$ $\mathrm{s}^{-1}$ during the expected night interval $(2100$ to $0900 \mathrm{~h})$ did not significantly reduce sporulation (Table 1 ), nor could sporulation above trace levels be induced during the expected day interval (0900 to 2100 h) by withholding light (Table 1). Similarly, when plants were conditioned to expect night to begin at $0900 \mathrm{~h}$,

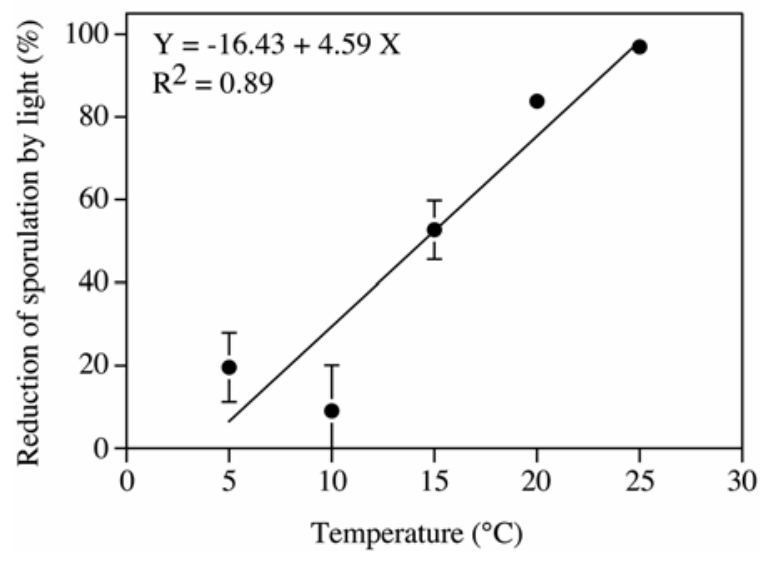

Fig. 4. Reduction of sporulation by light $\left(40 \mu \mathrm{mol} \mathrm{m} \mathrm{m}^{-2} \mathrm{~s}^{-1}\right)$ relative to dark controls in Bremia lactucae NY-1. Lettuce seedlings were incubated for $10 \mathrm{~h}$ at different temperatures and $100 \%$ relative humidity in light or darkness. Bars indicate one standard error of the mean at respective temperatures. Errors not visible approached 0 . 
exposure to light during the expected night interval (0900 to 2100 h) did not significantly reduce sporulation (Table 1 ), nor could sporulation above trace levels be induced during the expected day interval (2100 to 0900) by withholding light (Table 1).

Impact of timing of the period of high $\mathrm{RH}$ during long day lengths. ANOVA indicated no significant effect of experimental repeat $(P=0.05)$, and homogeneity of variance allowed data from the three repeats of the experiment to be pooled. Few spores were produced in seedlings grown in a 24-h light regime prior to incubation, regardless of the timing of the 4-h period of darkness and high RH (Table 2). In seedlings acclimated to a 3-h night, nil to slight sporulation was observed when the 4-h period of high

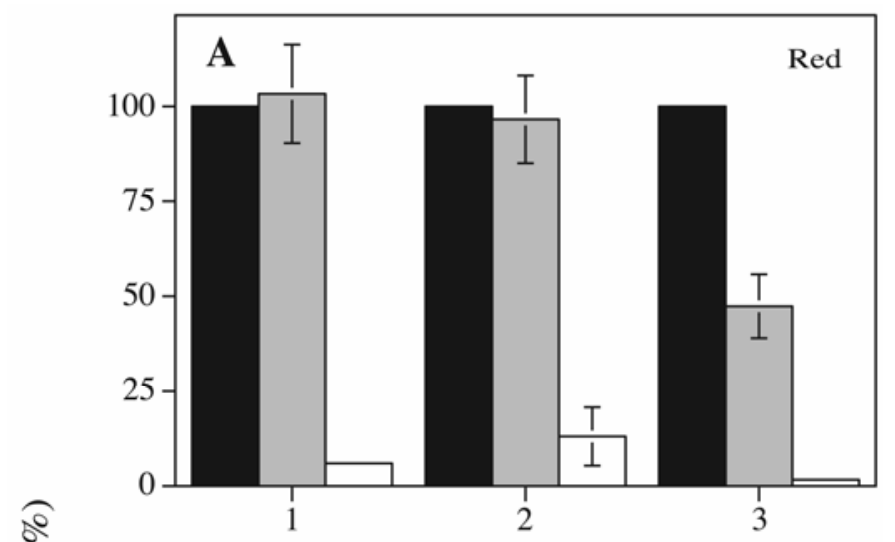

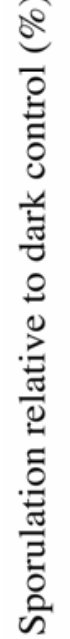
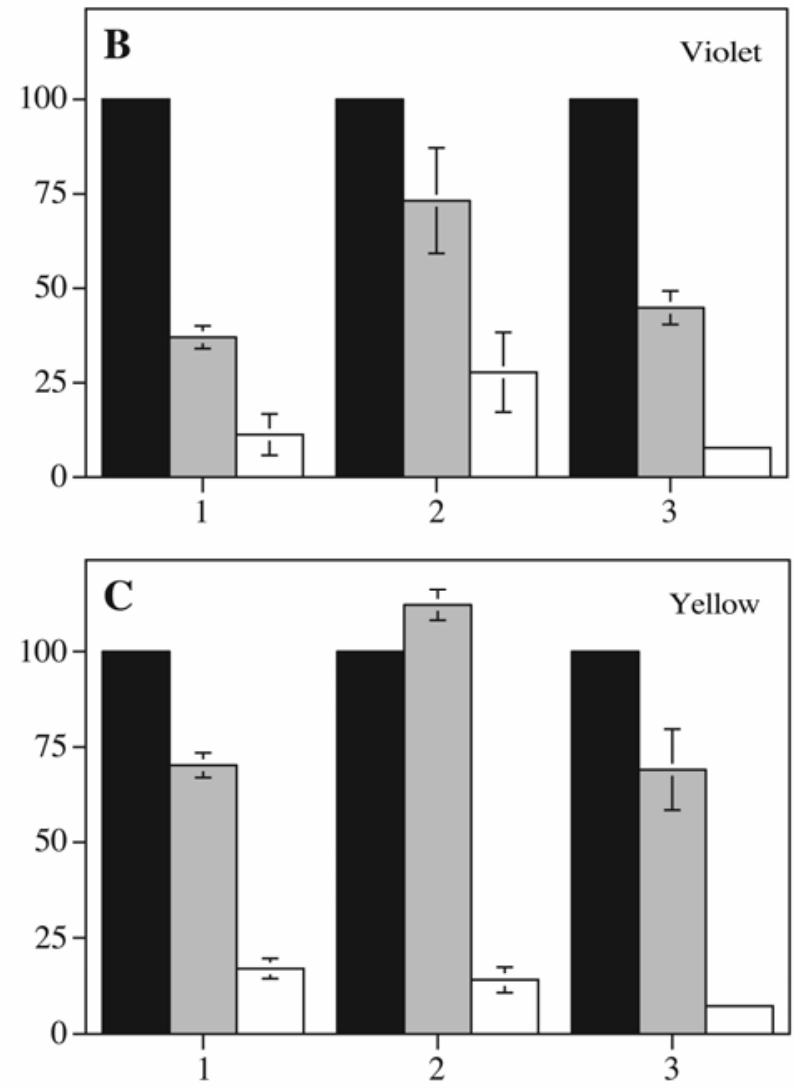

Experimental Repeat

Fig. 5. Effect of A, red; $\mathbf{B}$, violet and $\mathbf{C}$, yellow light (gray bars) provided through wide-bandpass filters on sporulation of Bremia lactucae NY-1 relative to dark controls (black bars) and daylight-balanced light (white bars). Detached lettuce cotyledons that previously were inoculated were incubated overnight at $20^{\circ} \mathrm{C}$ and $100 \%$ relative humidity. Intensity of daylight-balanced source was $13 \mu \mathrm{mol} \mathrm{m}{ }^{-2} \mathrm{~s}^{-1}$. Bars indicate standard error of the mean for each of three repetitions of the experiment.
$\mathrm{RH}$ began 1 or $0 \mathrm{~h}$, respectively, before the onset of darkness (Table 2), although sporophores developed, indicating that spores would have been formed if the period of high $\mathrm{RH}$ had been longer. Profuse sporulation occurred when the 4-h incubation period with high $\mathrm{RH}$ began 1 or $2 \mathrm{~h}$ after the onset of darkness.

Germination of spores produced in light and darkness. Spores produced in darkness had a significantly higher germination rate than spores produced in light at $20^{\circ} \mathrm{C}$ (i.e., $21.2 \pm 3.7 \%$ versus $2.3 \pm 1.5 \%$, respectively; mean \pm standard error). Generally, $>50 \%$ of spores produced in light were pigmented and atypically small (Fig. 8), similar to immature spores. None of these atypically smaller spores were observed to germinate.

\section{DISCUSSION}

Direct and interactive effects of light and temperature. Although our studies were conducted using only two isolates of B. lactucae, the temperature optimum for sporulation in our study was between 5 and $15^{\circ} \mathrm{C}$, which approximates the optimum temperature ranges reported by Powlesland (18) for isolates from the United Kingdom $\left(6\right.$ to $11^{\circ} \mathrm{C}$ ) and $\mathrm{Su}$ et al. (22) for isolates from California (10 to $20^{\circ} \mathrm{C}$ ). The suppressive effect of light on sporulation was strongly dependent upon temperature. Sporula-
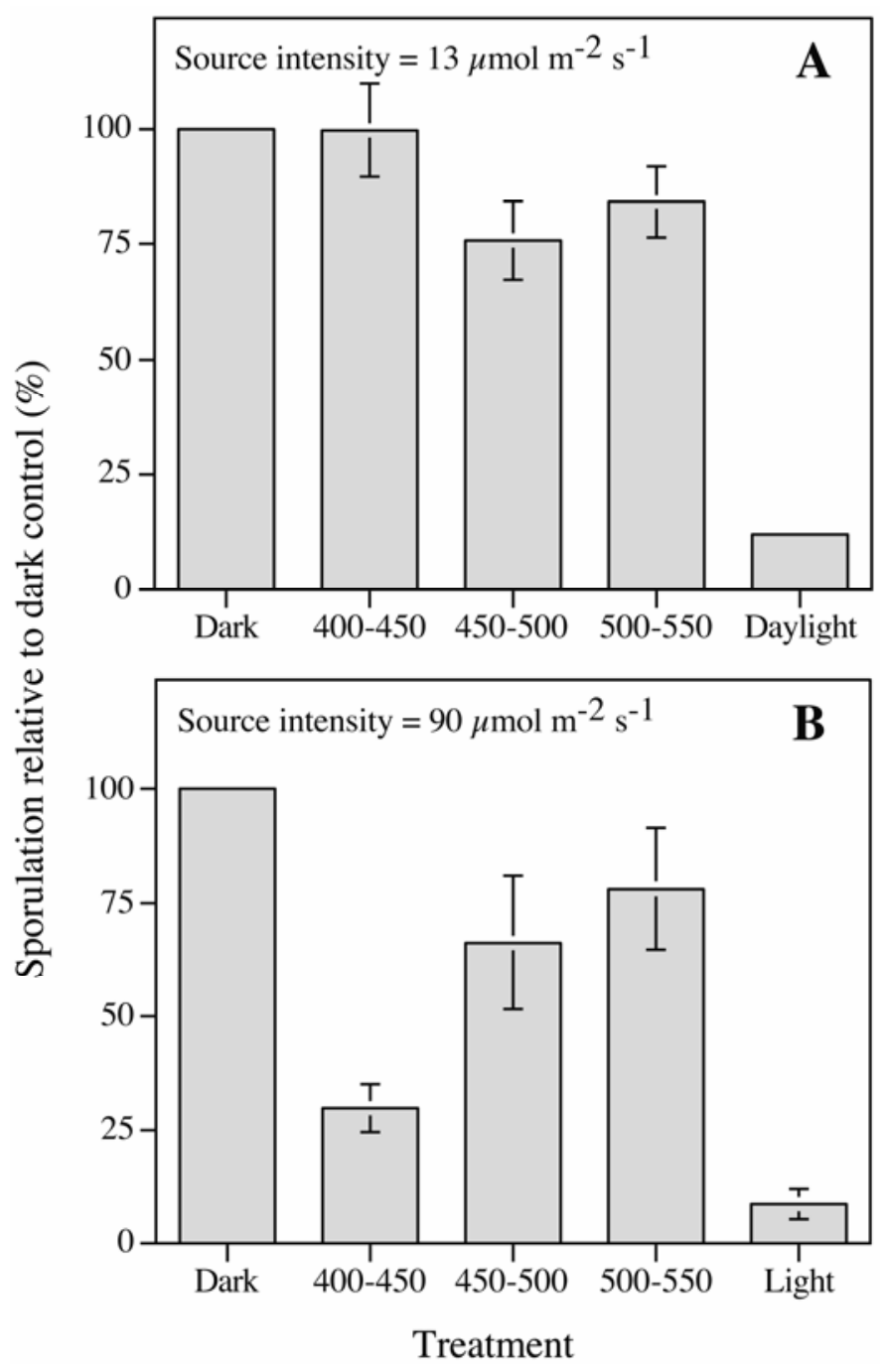

Fig. 6. Suppression of sporulation of Bremia lactucae A, NY-1 and B, NOR-1 by light provided through narrow-bandpass filters and daylight filter (NY-1) or unfiltered light (NOR-1) relative to dark controls. Detached lettuce cotyledons were incubated overnight at $20^{\circ} \mathrm{C}$ and $100 \%$ relative humidity. Intensity of the light source was $\mathbf{A}, 13$ or $\mathbf{B}, 90 \mu \mathrm{mol} \mathrm{m}^{-2} \mathrm{~s}^{-1}$. Bars indicate one standard error of the mean. Errors not visible approached 0 . 
tion of B. lactucae previously has been described as a dark-induced phenomenon where light is inhibitory $(19,25,27)$. However, all of the foregoing studies were conducted at temperatures in the range 15 to $22^{\circ} \mathrm{C}$, which is within the temperature range where light had the highest suppressive effect on sporulation in our experiments. An interaction of light and temperature has been reported for Phytophthora infestans (5) and Pseudoperonospora cubensis (4), where suppression of sporulation by blue light increased as temperature increased from 10 to $25^{\circ} \mathrm{C}$.

The existence of an interaction between temperature and the suppressive effect of light on sporulation obviously could affect the results of controlled environment studies of B. lactucae, and
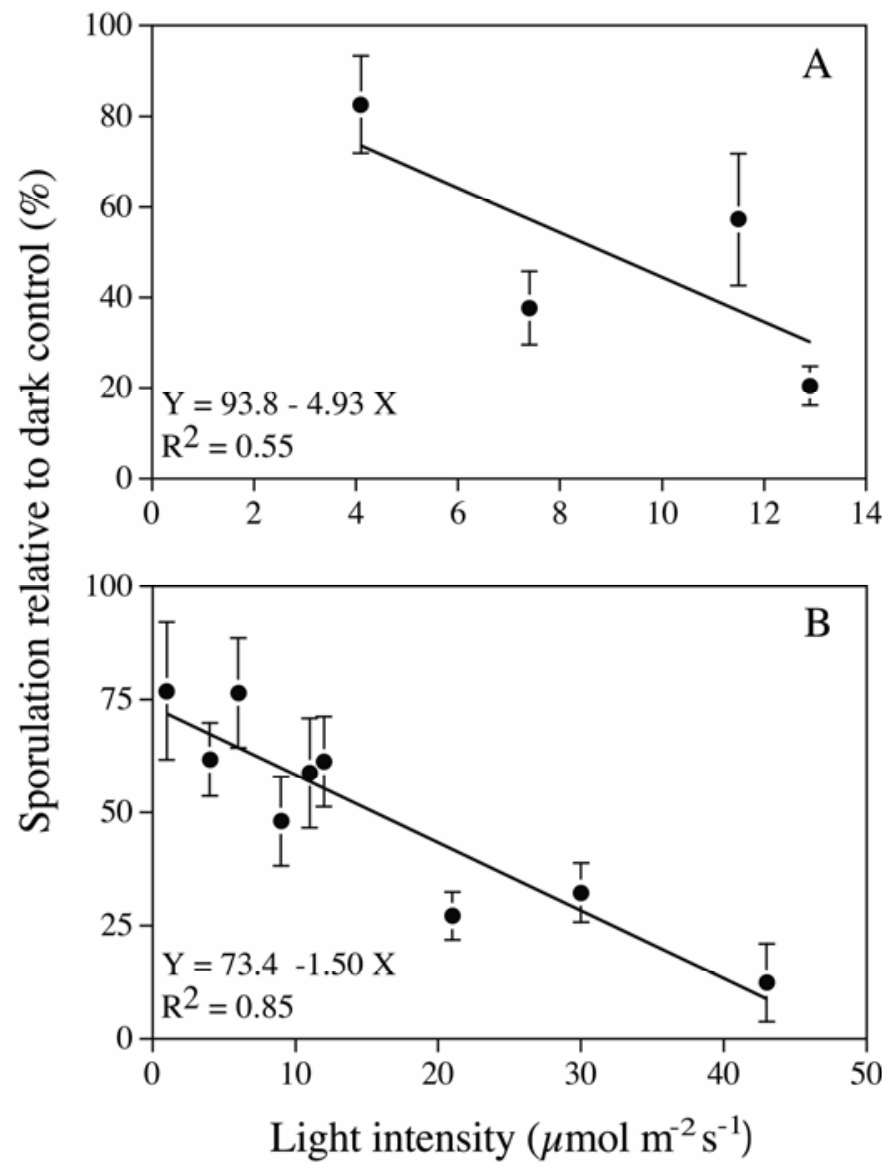

Fig. 7. Suppression of sporulation of Bremia lactucae A, NY-1 or B, NOR-1 at different light intensities relative to dark controls. Detached lettuce cotyledons were incubated overnight at $20^{\circ} \mathrm{C}$ and $100 \%$ relative humidity. Bars indicate one standard error of the mean at respective light intensities. possibly other downy mildews. Additionally, there are practical implications of the above interaction, whereupon night temperatures should be considered in forecasting the effect of light on sporulation. For example, in the lettuce-production areas near Lier, Norway, the average daily minimum temperature during the months of June and July is $\approx 10$ and $12{ }^{\circ} \mathrm{C}$, respectively (Bioforsk, Norway), and the suppressive effects of light on sporulation during extended twilight (e.g., 150 to $2 \mathrm{~W} \mathrm{~m}^{-2}$ at 2000 to $2300 \mathrm{~h}$ and 0300 to $0600 \mathrm{~h}$ at midsummer) (Norwegian University of Life Sciences) often would be largely negated due to low temperatures. Above-average night temperatures in such regions would increase the suppression of sporulation during extended twilight.

Our experiments were conducted on lettuce seedlings, which may be more susceptible to infections of $B$. lactucae than older seedlings and mature lettuce plants, as demonstrated by Dickinson and Crute (7). However, the interactive effects of light and temperature demonstrated in our experiments most likely are also the same for mature lettuce plants.

Effects of light intensity and quality of sporulation. At temperatures where light causes a suppressive effect on spore production, light in the violet range (400 to $450 \mathrm{~nm}$ ) was the component responsible for the greatest reduction in sporulation intensity in our studies. However, a minimum threshold of light intensity had to be reached to demonstrate this effect. Our findings contrast with the results of Raffray and Sequeira (19), who detected no inhibition of sporulation of B. lactucae in the blueviolet area at $18^{\circ} \mathrm{C}$, whereas only a few scattered sporophores, similar to seedlings exposed to unfiltered light, were developed in the green range of light (maximum transmission at $526 \mathrm{~nm}$ ). The intensity of light provided through the filters used by Raffray and Sequeira (19) was not presented. In preliminary studies (B. Nordskog, unpublished data), we found that violet wide-bandpass filters transmitted substantially less total light than green widebandpass filters $\left(5.5 \mu \mathrm{mol} \mathrm{m} \mathrm{m}^{-2} \mathrm{~s}^{-1}\right.$ versus $23.0 \mu \mathrm{mol} \mathrm{m} \mathrm{m}^{-2} \mathrm{~s}^{-1}$ using equivalent light sources). Thus, it is possible that the effect observed by Raffray and Sequeira (19) was due partially to light in-

TABLE 2. Sporulation of Bremia lactucae when incubated in darkness at $100 \%$ relative humidity $(\mathrm{RH})$ and $15^{\circ} \mathrm{C}$ for $4 \mathrm{~h}$ at different times of day following two different light regimes and four incubation periods ${ }^{\mathrm{a}}$

\begin{tabular}{lcccc}
\hline & \multicolumn{4}{c}{ Incubation period $^{\mathrm{b}}$} \\
\cline { 2 - 5 } Dark period & $0800-1200 \mathrm{~h}$ & $0900-1300 \mathrm{~h}$ & $1000-1400 \mathrm{~h}$ & $1100-1500 \mathrm{~h}$ \\
\hline $0 \mathrm{~h}$ & $0.3 \pm 0.3$ & $1.4 \pm 0.6$ & $3.3 \pm 1.9$ & $6.1 \pm 2.2$ \\
$3 \mathrm{~h}$ & $0.8 \pm 0.6$ & $1.1 \pm 0.6$ & $17.5 \pm 7.8$ & $26.7 \pm 10.0$ \\
\hline
\end{tabular}

${ }^{a}$ Prior to incubation, the seedlings were grown in growth chambers at $15^{\circ} \mathrm{C}$ and $70 \% \mathrm{RH}$ at two different light regimes of continuous light or $3 \mathrm{~h}$ of dark beginning at 0900 , respectively.

${ }^{\mathrm{b}}$ Mean number of spores $\left(10^{3}\right)$ harvested per cotyledon (mean \pm standard error).

TABLE 1. Effect of diurnal rhythm on sporulation of Bremia lactucae on seedlings incubated at $15^{\circ} \mathrm{C}$ and $100 \%$ relative humidity (RH) for $12 \mathrm{~h}$ in either light or darkness at different times of the day ${ }^{\mathrm{a}}$

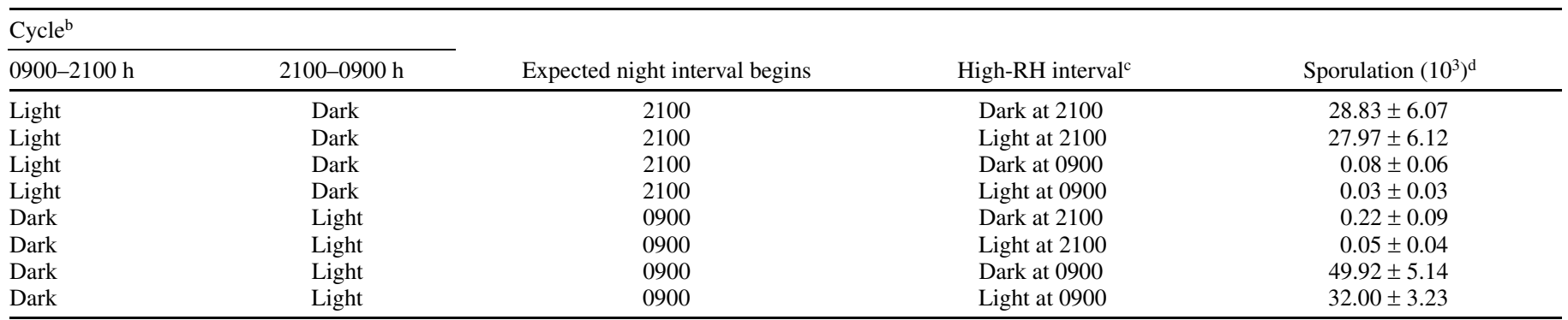

a Prior to incubation the seedlings were grown in growth chambers at $15^{\circ} \mathrm{C}$ and $70 \% \mathrm{RH}$ with two temporally offset light regimes, each providing $12 \mathrm{~h}$ of light from 0900 to 2100 and 2100 to 0900 , respectively.

${ }^{\mathrm{b}}$ Day and night cycle during seedling growth and incubation.

${ }^{c}$ Starting time of and illumination during interval of high RH.

${ }^{\mathrm{d}}$ Mean number of spores harvested per cotyledon \pm standard error. 
tensity rather than light quality. Likewise, the maximum inhibition of sporulation for Peronospora tabacina was obtained using a wide-bandpass filter transmitting wavelengths from 469 to $524 \mathrm{~nm}$ (4), using a wide-bandpass filter with a peak transmittance of $436 \mathrm{~nm}$ (20) for Plasmopara viticola, at both near-UV (310 to $400 \mathrm{~nm}$ ) and green (500 to $560 \mathrm{~nm}$ ) for P. viticola (2), and in the blue range (peak intensity at $450 \mathrm{~nm}$ ) and green range (peak intensity at $550 \mathrm{~nm}$ ) for Pseudoperonospora cubensis (4). Our results indicate that both source light intensity and quality, as well as the spectral distribution and transmittance of any filters used, should be considered before specific wavebands are definitively identified as inhibitory to sporulation. Wide-bandpass filters can transmit substantial radiant energy beyond the peak transmittance (Fig. 1), and the reduction of energy inherent in the use of narrow-bandpass filters (Fig. 2) may require substantial increases in source intensity to demonstrate a treatment effect if a threshold quantum of light is required to suppress sporulation. The quantum meters used in our studies measure radiation within the photosynthetic active radiation spectrum (400 to $700 \mathrm{~nm}$ ), whereas radiation at wavelengths $>700$ and $<400 \mathrm{~nm}$ was not measured. Therefore, we have no knowledge whether light in the IR or UV spectrum could have had additive effects on sporulation of $B$. lactucae. However, the suppressive effect of violet light on sporulation indicates that light in the blue range and near-UV light represents the wavelengths with the greatest inhibitory effect on sporulation of B. lactucae in vitro.

In our experiments, the suppressive effect of white light on sporulation of $B$. lactucae was highly variable at the lowest light intensity tested $\left(1 \mu \mathrm{mol} \mathrm{m} \mathrm{m}^{-2} \mathrm{~s}^{-1}\right)$, but it stabilized at light intensities $>10 \mu \mathrm{mol} \mathrm{m} \mathrm{m}^{-2} \mathrm{~s}^{-1}$. Wu et al. (26) inferred a photon flux density of $41 \mu \mathrm{mol} \mathrm{m} \mathrm{m}^{-2} \mathrm{~s}^{-2}$ as stimulatory to spore release from field observations of leaf wetness and subsequent disease development; however, we are not aware of previous studies that would indicate a threshold level of light that would suppress sporulation in B. lactucae. Our results indicate that light intensities considerably lower than levels previously used to study the release of extant spores $(22,26)$ will suppress sporulation when temperatures are $>20^{\circ} \mathrm{C}$, and that failure to control or account for the possible interactive effects of temperature and diurnal periodicity could produce seemingly disparate effects of light on sporulation of B. lactucae.

Effect of diurnal rhythm on sporulation. Nocturnal sporulation in some downy mildews has been reported to be influenced by diurnal rhythms (27). In our experiments, sporulation of B. lactucae occurred during an expected night interval regardless of the presence of light or darkness. This indicates that a circadian rhythm can operate independently of light in the timing of onset of sporulation. Because B. lactucae is an obligate parasite, it is not possible to distinguish effects caused by the host and the pathogen and thereby determine whether circadian rhythms in the plant or the pathogen, or a combination of these factors, control timing of sporulation in B. lactucae. However, circadian clocks have been shown to control daily rhythms of spore development and discharge for several fungi (1). Sporulation and subsequent release often appear as a diurnal rhythm and are associated with diurnal changes in humidity, temperature, air turbulence, and light. Initial spore release of $B$. lactucae has been reported to coincide with sunrise under field conditions $(3,21)$. Su et al. (22) associated trapping of spores in a controlled-environment system with exposure to a light intensity of $74 \mu \mathrm{mol} \mathrm{m} \mathrm{m}^{-2}$ although, in the absence of light and dark control treatments, it is unclear whether diurnal periodicity affected spore release in their experiments. Attribution of spore release to the foregoing environmental factors from field data possibly has obscured a more prominent causal role for circadian rhythms in sporulation of downy mildews. Of particular concern should be the possible confounding of results from controlled-environment studies if normal circadian rhythms are not synchronous with light and dark cycles.
Impact of timing of the period of high RH during long day lengths. Although, Raffray and Sequeira (19) suggested that the development of the B. lactucae could be completely inhibited by continuous light, this was not the case in our studies. Rumbolz et al. (20) reported for Plasmopara viticola that continuous white light did not affect formation of sporangiophores, but sporangia that developed in continuous light remained immature. However, a brief dark period following lengthy incubation in white light resulted in production of a few sporangiophores with mature sporangia. This corresponds with our results, where a few spores were produced after incubation in darkness following an extended period of continuous light. Verhoeff (25) reported that darkness prior to the onset of high $\mathrm{RH}$ reduced the time required for sporulation. We found profuse sporulation in seedlings grown at a 3-h night interval when incubation at high RH occurred after the onset of darkness, indicating that the onset of the sporulation period started at this time.

Germination of spores produced in light and darkness. Light not only reduced the quantity of spores produced by $B$. lactucae but, in addition, the germination rate of spores produced in light was $\approx 1 / 10$ that of spores produced in darkness. Reduced germination of light-produced spores was associated with their overall smaller size and occasional darkened appearance (Fig. 8). Our results are in accordance with those of Rumbolz et al. (20), who found that sporangiophores of $P$. viticola which developed in light failed to produce completely differentiated sporangia. Also, light is likely to have delayed the onset of sporulation.

Practical impact of light, temperature, and diurnal rhythms in forecasting systems for downy mildew on lettuce. Current forecasting models for lettuce downy mildew use sunrise and sunset to delimit the hours during which leaf wetness and high $\mathrm{RH}$ can induce sporulation. In the lettuce-growing areas of Nor-

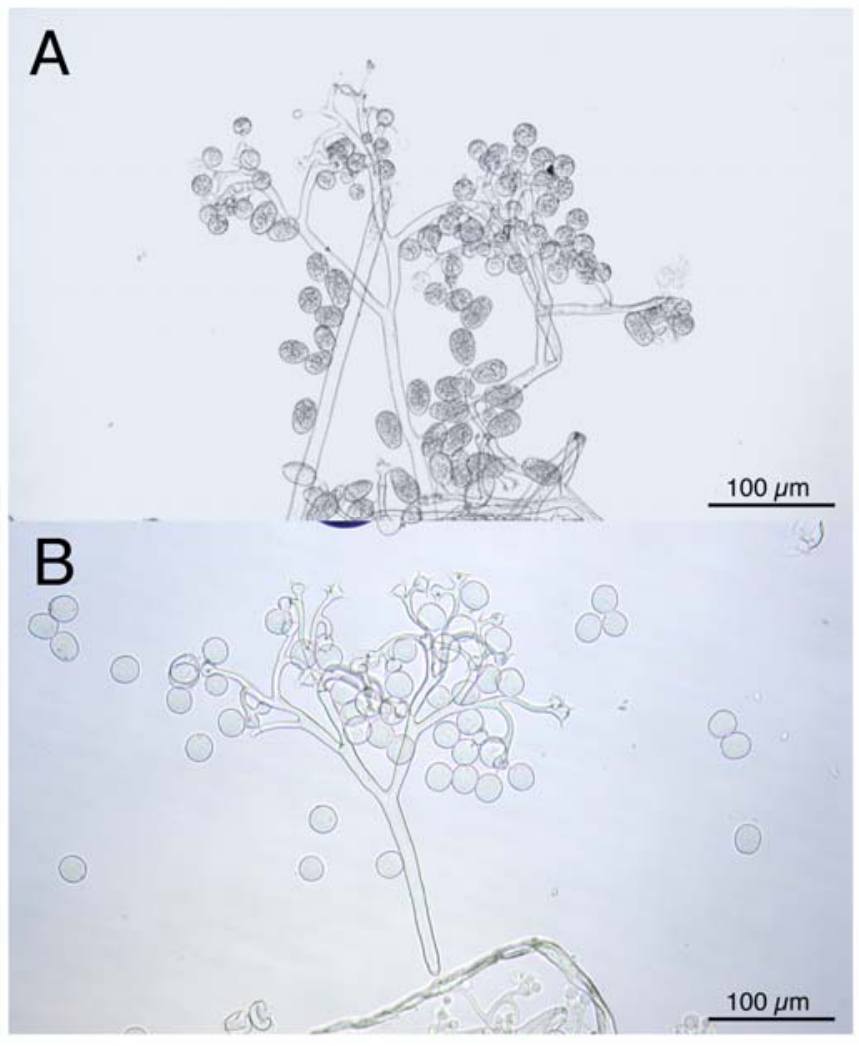

Fig. 8. A, Sporophore and spores of Bremia lactucae NY-1 produced during exposure to daylight-balanced illumination at $13 \mu \mathrm{mol} \mathrm{m} \mathrm{m}^{-2} \mathrm{~s}^{-1}$ showing darkened appearance and reduced spore size compared with $\mathbf{B}$, sporophore and spores of $B$. lactucae NY-1 produced in darkness. 
way and other countries at high latitudes it is important to incorporate the possible effects of short nights and extended twilight conditions into forecasting models. Additionally, temperature should be used to modify model predictions related to sporulation, because the suppressive effect of light on sporulation is temperature dependent and may be greatly reduced at temperatures $<15$ to $20^{\circ} \mathrm{C}$. Finally, although we have demonstrated a diurnal rhythm in $B$. lactucae, it remains unclear how diurnal rhythms might interact with light and temperature under field conditions to affect sporulation and dispersal of spores. At temperatures below the level at which light might suppress sporulation, our experiments indicate that the diurnal rhythm is a controlling factor in timing of sporulation. It is noteworthy that, despite variation in time of sunrise, day length, and environmental conditions, peak airborne concentrations of spores of B. lactucae often occur near 1100 h (3,21; B. Nordskog, unpublished data), long after sunrise, perhaps reflecting both a diurnal rhythm and atmospheric conditions more favorable to airborne dispersal of spores.

\section{ACKNOWLEDGMENTS}

This work was funded by the Bioforsk-Norwegian Institute for Agricultural and Environmental Research, The Research Council of Norway, Innovation Norway, and Norwegian lettuce growers.

\section{LITERATURE CITED}

1. Bell-Pedersen, D., Garceau, N., and Loros, J. J. 1996. Circadian rhythms in fungi. J. Genet. 75:387-401.

2. Brook, P. J. 1979. Effect of light on sporulation on Plasmopara viticola. N.Z. J. Bot. 17:135-138

3. Carisse, O., and Philion, V. 2002. Meteorological factors affecting periodicity and concentration of airborne spores of Bremia lactucae. Can. J. Plant Pathol. 24:184-193.

4. Cohen, Y., and Eyal, H. 1977. Growth and differentiation of sporangia and sporangiophores of Pseudoperonospora cubensis on cucumber cotyledons under various combinations of light and temperature. Physiol. Plant Pathol. 10:93-103.

5. Cohen, Y., Eyal, H., and Sadon, T. 1975. Light-induced inhibition of sporangial formation of Phytophthora infestans on potato leaves. Can. J. Bot. 53:2680-2686.

6. Cruickshank, I. A. M. 1963. Environment and sporulation in phytopathogenic fungi. IV. The effect of light on the formation of conidia of Peronospora tabacina Adam. Aust. J. Biol. Sci. 16:88-98.

7. Dickinson, C. H., and Crute, I. R. 1974. The influence of seedling age and development on the infection of lettuce by Bremia lactucae. Ann. Appl. Biol. 76:49-61.

8. Fletcher, J. 1976. Bremia lactucae, oospores, sporangial dissemination and control. Ann. Appl. Biol. 84:294-298.

9. Gadoury, D. M., Stensvand, A., and Seem, R. C. 1996. A wind tunnel for controlled-environment studies of ascospore release by Venturia inaequalis. Phytopathology 86:596-601.

10. Gilles, T., Phelps, K., Clarkson, J. P., and Kennedy, R. 2004. Development of MILIONCAST, and improved model for predicting downy mildew sporulation on onions. Plant Dis. 88:695-702.

11. Jesperson, G. D., and Sutton, J. C. 1987. Evaluation of a forecaster for downy mildew on onion (Allium cepa $\mathrm{L}$.). Crop Prot. 6:95-103.

12. Leach, C. M., Hildebrand, P. D., and Sutton, J. C. 1982. Sporangium discharge by Peronospora destructor: Influence of humidity, red-infrared radiation, and vibration. Phytopathology 72:1052-1056.

13. Madden, L. V., Ellis, M. A., Lalancette, N., Hughes, G., and Wilson, L. L. 2000. Evaluation of a disease warning system for downy mildew of grapes. Plant Dis. 84:549-554.

14. Magarey, P. A., Wachtel, M. F., Weir, P. C., and Seem, R. C. 1991. A computer-based simulator for rational management of grapevine downy mildew (Plasmopara viticola). Plant Prot. Q. 6:29-33.

15. Nordskog, B., Gadoury, D. M., Seem, R. C., and Hermansen, A. 2005 Effects of light intensity and quality on sporulation of lettuce downy mildew (Bremia lactucae). (Abstr.) Page 58 in: Proc. 9th Int. Workshop Plant Dis. Epidemiol. Landerneau, France.

16. Nordskog, B., Hermansen, A., Seem, R. C., and Gadoury, D. M. 2004. Evaluation of two models for lettuce downy mildew infection periods in Norway. (Abstr.) Phytopathology 94:S76.

17. Park, E. W., Seem, R. C., Gadoury, D. M., and Pearson, R. C. 1997. DMCast: A prediction model for grape downy mildew development. Vitic. Enol. Sci. 52:182-189.

18. Powlesland, R. 1954. On the biology of Bremia lactucae. Trans. Br. Mycol. Soc. 37:362-371.

19. Raffray, J. B., and Sequeira, L. 1971. Dark induction of sporulation in Bremia lactucae. Can. J. Bot. 49:237-239.

20. Rumbolz, J., Wirtz, S., Kassemeyer, H.-H., Guggenheim, R., Schäfer, E., and Büche, C. 2002. Sporulation of Plasmopara viticola: Differentiation and light regulation. Plant Biol. 4:413-422.

21. Scherm, H., and van Bruggen, A. H. C. 1995. Concurrent spore release and infection of lettuce by Bremia lactucae during mornings with prolonged leaf wetness. Phytopathology 85:552-555.

22. Su, H., van Bruggen, A. H. C., and Subbarao, K. V. 2000. Spore release of Bremia lactucae on lettuce is affected by light initiation and decrease in relative humidity. Phytopathology 90:67-71.

23. Su, H., van Bruggen, A. H. C., Subbarao, K. V., and Scherm, H. 2004. Sporulation of Bremia lactucae affected by temperature, relative humidity, and wind in controlled conditions. Phytopathology 94:396-401.

24. Thomas, B., and Vince-Prue, D. 1997. Photoperiodism in Plants. Academic Press, London.

25. Verhoeff, K. 1960. On the parasitism of Bremia lactucae Regel on lettuce. Tijdschr. Plantenziekten 66:133-203.

26. Wu, B. M., van Bruggen, A. H. C., Subbarao, K. V., and Scherm, H. 2002. Incorporation of temperature and solar radiation thresholds to modify a lettuce downy mildew warning system. Phytopathology 92:631-636.

27. Yarwood, C. E. 1937. The relation of light to the diurnal cycle of sporulation of certain downy mildews. J. Agric. Res. 54:365-373. 